\title{
Pregnant-Postpartum Women with Severe Epigastric Pain and HELLP Syndrome: A Highly Dangerous Combination
}

\author{
James Nello Martin Jr.*, James Martin Tucker \\ Department of Obstetrics and Gynecology, The Winfred L. Wiser Hospital for Women \& Infants at the University of Mississippi \\ Medical Center, Jackson, Mississippi, USA \\ Email: *jnmartinjrmd@gmail.com
}

How to cite this paper: Martin Jr., J.N. and Tucker, J.M. (2021) Pregnant-Postpartum Women with Severe Epigastric Pain and HELLP Syndrome: A Highly Dangerous Combination. Open Journal of Obstetrics and Gynecology, 11, 1081-1091.

https://doi.org/10.4236/ojog.2021.118101

Received: July 22, 2021

Accepted: August 27, 2021

Published: August 30, 2021

Copyright $\odot 2021$ by author(s) and Scientific Research Publishing Inc. This work is licensed under the Creative Commons Attribution International License (CC BY 4.0).

http://creativecommons.org/licenses/by/4.0/

(c) (i) Open Access

\begin{abstract}
OBJECTIVE: HELLP syndrome is a severe preeclampsia spectrum disorder diagnosed when laboratory evidence of hemolysis, liver dysfunction and thrombocytopenia are present. The presence of epigastric pain with laboratory criteria for HELLP syndrome may indicate higher maternal-fetal risk. Thus we explored maternal outcomes in 42 pregnant/postpartum women that had HELLP syndrome by laboratory criteria in addition to sudden severe epigastric pain. METHODS: A database was constructed from the medical files of all patients with medical/hypertensive disorders evaluated by the first author from 1986-2015 for medicolegal purposes. All patient files of women who presented to their physicians with a diagnosis of presumptive HELLP syndrome were examined. RESULTS: Fifty-eight pregnant/postpartum women fulfilled study inclusion criteria; they presented to physicians for evaluation usually in non-tertiary care hospital settings. Clinical presentation and care including lab data and details of pregnancy outcome were evaluated. A correct diagnosis of HELLP syndrome was verified for 42 women (72.4\%); others were determined to have AFLP-Acute Fatty Liver of Pregnancy $(\mathrm{n}=8)$ or TTP-aHUS-Thrombotic thrombocytopenic purpura-adult/atypical hemolytic uremic syndrome $(\mathrm{n}=8)$. All 42 HELLP women $(100 \%)$ in our cohort had severe epigastric pain. Treatment modalities for maternal HELLP syndrome included magnesium sulfate $(\mathrm{n}=35,83 \%)$, antihypertensives $(\mathrm{n}=17,40 \%)$; corticosteroids (CORT) for HELLP were not utilized. Major maternal morbidity (21 strokes, 9 liver ruptures/hematomas) affected 41 HELLP women (98\%); 22 died, 7 were permanently disabled. CONCLUSION: HELLP syndrome patients presenting with sudden, severe epigastric pain in this highly selected cohort experienced very high maternal morbidity and mortality. Laboratory evidence of HELLP syndrome in association with epigastric pain is a
\end{abstract}


dangerous combination that portends great danger to safe motherhood. We speculate that the absence of CORT use in this cohort contributed to poor maternal outcome (word count $=280$ as modified for reviewers).

\section{Keywords}

HELLP SYNDROME with or without Epigastric Pain, Maternal Morbidity \& Mortality, LDH to AST Ratio

\section{Introduction}

Four decades ago, Dr. Louis Weinstein proposed the acronym "HELLP syndrome" to characterize a special group of pregnant or postpartum patients with a severe preeclampsia-spectrum disorder characterized by laboratory evidence of hemolysis, elevated liver enzymes and moderate to severe thrombocytopenia [1]; epigastric pain was not a criterion then for the diagnosis nor had it become a criterion in the intervening years (ACOG 2013, 2020). Over time controversy about HELLP has persisted with regard to diagnostic criteria [2] [3], systems of classification [4] [5] [6], and recommendations for treatment [7] [8] [9] [10].

As a unique and infrequent form of preeclampsia, HELLP presents a diagnostic and therapeutic challenge to clinicians and a challenge also to investigators wanting to undertake large randomized prospective clinical research trials. To date there has not been a large multicenter trial in the United States to rigorously study various proposed treatments for HELLP syndrome patients such as potent glucocorticoids (CORT), especially with stratification of results based upon composite severity of disease expression such as comparing patients with severe epigastric pain to those with milder asymptomatic, "laboratory abnormality only" HELLP syndrome. Almost four decades after the acronym HELLP was formalized, the most recent meta-analysis of worldwide prospective trials comparing potent corticosteroids (CORT) versus matched controls as principal therapy for HELLP was able to aggregate only 22 qualifying studies [11] [12]. These were not separated for analysis according to the presence or absence of severe epigastric pain in the study subjects.

Novel research methodologies such as maternal morbidity/mortality reviews that aggregate statewide and national data of infrequently occurring conditions, or aggregations of medicolegal case analyses that are properly deidentified to protect patient confidentiality, can be used to assess the impact of various therapies on diseases and disorders notwithstanding their inherent risk of selection bias. Both of these approaches to the study of infrequently occurring specific maternal complications or diseases can provide worthwhile information both for clinicians and researchers.

Our purpose in this retrospective investigation was to explore maternal-fetal outcomes in a large previously unreported 1986-2015 cohort of women with presumptive HELLP syndromes that were managed by obstetrician-gynecologists 
practicing mostly in diverse hospital settings in the United States which thereafter led to litigation related to causation and appropriateness of rendered care. Maternal-perinatal outcomes were assessed with regard to accomplishing timely and safe delivery employing various therapies that have been advocated for women with this disorder.

\section{Materials and Methods}

Over three decades between 1986 and 2015 the first author was independently retained to critically evaluate for medicolegal defense purposes 161 medicolegal files of patients that experienced medical or hypertensive disorders of pregnancy. After all legal activities involving each case were concluded, all legal documents and communications were shredded and only the de-identified medical record materials were retained in locked cabinets for possible later review, study and/or use in teaching exercises. Among the 161 confidential, privately maintained and HPPA-compliant protected medical record files were 58 women that had a probable diagnosis of presumptive HELLP syndrome at initial presentation; each was reviewed for possible inclusion in this investigation. Subsequently 8 women were determined to have AFLP (acute fatty liver of pregnancy) and 8 others had either TTP (thrombotic thrombocytopenic purpura) or adult/atypical HUS (hemolytic uremic syndrome); the findings with these 16 women have been published previously and are not addressed in the present report [13] [14].

Following detailed file review and data extraction from the remaining 42 study subjects that met HELLP diagnostic criteria, a clinical summary for each woman was prepared including details of presentation, clinical findings, lab results, surgeries, interventions, complications, and maternal-fetal/newborn outcomes. Careful attention was given to details of the three principal therapeutic interventions proposed for patients with HELLP syndrome: magnesium sulfate by infusion, antihypertensive therapy to minimize severe systolic hypertension, and corticosteroid (CORT) administration either for fetal and/or maternal indication(s) [15]. In some instances there was enough data to accurately determine a time course between onset of some signs/symptoms and later outcomes. Data extraction accuracy was verified by both authors; no other persons had access to these confidential medical records.

A diagnosis of HELLP syndrome was made in the presence of $\mathrm{HE}=$ hemolysis (microangiopathic hemolytic anemia reflected by elevated indirect bilirubin $\geq$ $1.2 \mathrm{mg} / \mathrm{dL}$, schistocytes on peripheral smear, haptoglobin $<25$, or total serum $\mathrm{LDH} \geq 600 \mathrm{IU} / \mathrm{L}), \mathrm{EL}=$ elevated liver enzymes (AST and/or ALT $\geq 70 \mathrm{IU} / \mathrm{L}$ or above twice normal values), and LP $=$ low platelets (platelets $\leq 100,000 /$ ul) [2] [3]. Patients were classified as having severe thrombocytopenia/Class 1 HELLP with platelet nadir $\leq 50,000 / \mathrm{ul}$, moderate thrombocytopenia/Class 2 HELLP with platelet nadir $>50,000 /$ ul but $\leq 100,000 /$ ul, and mild thrombocytopenia/Class 3 HELLP with platelet nadir $>100,000 /$ ul but $\leq 150,000 /$ ul [4] [5]. Presence or absence of symptomatology including epigastric pain was recorded. 
Categories of major maternal morbidity included intracranial hemorrhage (ICH), cerebral edema, cerebral venous thrombosis (CVT), eclampsia/eclampsia like convulsion, sustained severe systolic hypertension (SSH), placental abruption, acute pulmonary edema, acute kidney injury (AKI), acute liver rupture/hemorrhage, disseminated intravascular coagulation (DIC), cardiac dysfunction or failure, and indicated blood/plasma/platelet transfusion. Once the initial reviews and data sheets were completed for each study subject, simple descriptive basic statistical analysis was undertaken; the information was reviewed confidentially by the second author to assure accuracy of the recorded findings.

\section{Results}

Comprehensive medical information was present for all 42 study subjects. Mean maternal age was 29 years (range 16 - 43 years); $62 \%$ were nulliparas, $38 \%$ were parous. There were 25 White, 11 Black, 1 Asian and 4 Hispanic women. Mean gestational age at delivery was 34 2/7ths weeks (range 23 - 40 3/7ths); 21 were $\leq 34$ weeks' gestation at the time of hospital admission. All 42 women in the cohort (100\%) experienced epigastric pain usually described as severe and sudden in onset. Laboratory testing obtained immediately after epigastric pain was first reported revealed a mean platelet count of 122,400/ul (range 67,000 - 192,000/ul) and a mean AST level of $315 \mathrm{IU} / \mathrm{L}$ (range $31-2771 \mathrm{IU} / \mathrm{L}$ ). Subsequently the platelet count nadired in the Class 1 HELLP range of $\leq 50,000 / \mathrm{uL}$ in 29 women and in the Class 2 HELLP range of $>50,000-\leq 100,000 /$ uL for 9 women; classification was indeterminate in four.

As shown in the accompanying Table 1, other laboratory findings included a mean peak AST level of 1461 IU/L (range 73 - 3902 IU/L). Total serum LDH levels were obtained by the treating physicians in only 18 of the 42 HELLP women, revealing a mean peak level of $3061 \mathrm{IU} / \mathrm{L}$ (range 341 - 11,796 IU/L); highest levels occurred in 9 HELLP women shown to have liver hematoma or rupture. Inclusion of LDH assessments as a component of laboratory screening and surveillance of patients with presumptive HELLP syndrome was requested in less than half of the HELLP cohort ( $\mathrm{n}=18,43 \%$ ), thus revealing a frequent physician shortcoming of not documenting evidence of the "HE" portion for diagnosing hemolysis/microangiopathic hemolytic anemia ( $>600 \mathrm{IU} / \mathrm{L}$ ) if a haptoglobin of $<25$, indirect bilirubin of $>1.2$ or evidence of schistocytes were not assessed [2]. Consistent with prior reports showing that patients with HELLP syndrome as opposed to those with TTP/aHUS exhibit low LDH to AST calculated ratios, the mean LDH to AST ratio for our cohort was 2.1 to 1 [16] [17].

Magnesium sulfate was utilized for 35 of the 42 HELLP women in the study cohort (83\%); in 3 others it was begun after eclamptic convulsion or other complication(s) developed. Antihypertensives were utilized in 17 women (40\%; 51\% of 33 with severe systolic hypertension or SSH) without achieving effective blood pressure control as assessed against ACOG guidelines in place since 2011 [18]. CORT was not a component of HELLP therapy for any women in our cohort. 
Table 1. Data summary for HELLP patient cohort.

\begin{tabular}{|c|c|}
\hline \multicolumn{2}{|c|}{ DELIVERY YEARS 1986-2015 } \\
\hline HELLP SYNDROME $(\mathrm{n}=42)$ & \\
\hline Maternal Age (years) & $29(16-43)$ \\
\hline Gestational Age (weeks) & $342 / 7$ \\
\hline Race (\# White/\# Black) & $25 / 11$ \\
\hline Parity (\% Nulliparity) & $62 \%$ \\
\hline Epigastric Pain (\%) & $100 \%$ \\
\hline RX: Magnesium Sulfate & $83 \%$ \\
\hline RX: Antihypertensives & $41 \%$ \\
\hline RX: CORTicosteroids & $0 \%$ \\
\hline \multicolumn{2}{|l|}{ MAJOR MATERNAL MORBIDITY } \\
\hline CNS: Stroke/ICH & $50 \%$ \\
\hline LIVER: Bleed/Rupture & $21 \%$ \\
\hline Severe Systolic HTN (SSH) & $79 \%$ \\
\hline Acute Pulmonary Edema & $52 \%$ \\
\hline Acute Kidney Injury (AKI) & $33 \%$ \\
\hline DIC & $33 \%$ \\
\hline \multicolumn{2}{|l|}{ LABORATORY INDICES } \\
\hline Mean Admission AST IU/L & $315(31-2771)$ \\
\hline Mean Admission PLTs/uL & $122,400 / \mathrm{uL}$ \\
\hline Mean Admission LDH IU/L & $514(143-1568)$ \\
\hline Mean Admission LDH:AST & $1.6: 1$ \\
\hline Peak AST IU/L & $1461(73-3902)$ \\
\hline Peak LDH IU/L & $3061(344-11,796)$ \\
\hline Peak LDH:AST Ratio & $2.1: 1$ \\
\hline \multicolumn{2}{|l|}{ MATERNAL OUTCOMES } \\
\hline Maternal Deaths & $22 / 42(52.4 \%)$ \\
\hline Maternal Disability & $7 / 42(16.7 \%)$ \\
\hline Maternal Intact Survival & $13 / 42(31 \%)$ \\
\hline
\end{tabular}

Means for groups are shown with ranges in parentheses.

Only 8 women among 23 of those eligible for indicated fetal lung maturation (FLM) steroids (undelivered, $\leq 34$ weeks' gestation, not requiring immediate delivery) received these medications; the last dose of FLM steroids was received $\geq 12$ hours to almost 4 days preceding the eventual onset of epigastric pain. Seven women late in the course of care for HELLP syndrome received either maintenance prednisone for systemic lupus erythematosus (SLE)/lupus anticoagulant ( $\mathrm{n}$ $=2$ ) or postpartum high dose corticosteroids (dexamethasone) to treat cerebral hemorrhage/cerebral edema formation. 
Major maternal morbidity affected $41(97 \%)$ women in this cohort. Half $(21 / 42,50 \%)$ suffered an ICH; 4 others developed cerebral edema from hypotension/shock events or eclamptic convulsion. In addition to the 33 women that sustained SSH, 22 had acute pulmonary edema (52\%), 14 developed encephalopathy (33\%), 14 had acute kidney injury/AKI (33\%), 14 had evidence of disseminated intravascular coagulation (DIC; 33\%), 10 had placental abruptions (24\%) and 9 had liver hematoma/ruptures (21\%) (see Table 1). Platelet counts obtained immediately preceding detection of a liver rupture/hematoma in the 9 affected women ranged between 167,000/ul and 17,000/ul, always in association with epigastric pain. Two women had shock-induced hypoxic brain and multiple organ injury, one had sepsis and one had uterine rupture. Transfusion was required in 31 of 38 patients (82\%) or 31 of $42(74 \%)$ for the entire cohort; chart information was missing to fully assess this factor in four members of the HELLP cohort.

Mode of delivery was cesarean in 31 (74\%); 6 of 8 women with postpartum-onset HELLP syndrome delivered vaginally as did 3 women with stillbirths in the antepartum-onset group. There were 7 stillbirths and 1 early neonatal death due to extreme prematurity. Maternal outcome was death for 22 mothers (52\%), permanent disability for 7 others (17\%), and 13 (31\%) survived the HELLP syndrome pregnancy medically intact.

\section{Discussion}

Almost four decades after the first report of the acronym "HELLP Syndrome" to facilitate identification and improved treatment of mothers suffering this potentially morbid or mortal complication of pregnancy, debate continues regarding optimal therapy for these women. A substantial number of women with "laboratory evidence only" of HELLP syndrome do not develop epigastric pain; they usually recover spontaneously following delivery/uterine evacuation and the provision of excellent medical (prevention of sustained severe systolic hypertension) and obstetric (timed delivery, magnesium sulfate, FLM steroids for preterm pregnancy) care. Most published patient series do not specify if epigastric pain were present or absent nor is data analyzed according to the presence or absence of this important reflector of disease severity. Because for many decades in the United States it has been taught that the presence of epigastric pain suggests the presence of HELLP syndrome until proven otherwise, the physicians caring for many of these study subjects obtained HELLP laboratory studies. Based on the very high major maternal morbidity and maternal mortality experienced by the 42 women with HELLP syndrome in this cohort, all of whom presented with severe epigastric pain, it seems likely that the presence of this significant symptom identifies a subset of HELLP patients who are at much greater risk of morbidity and mortality compared to asymptomatic HELLP patients.

We emphasize that $100 \%$ of our HELLP cohort experienced sudden, severe 
epigastric pain consistent with an acute attack on maternal hepatic structure/function. This was uniformly followed by rapidly deteriorating laboratory indices reflecting HELLP syndrome progression usually to merit classification as class 1 HELLP disease. Importantly, not all patients evidenced full criteria for HELLP syndrome at the time of epigastric pain onset but it always followed soon thereafter. Unless epigastric pain historically was present for longer than 4 hours prior to admission in our patient cohort, the initial platelet level obtained was usually not yet below 100,000/uL although AST was $\geq 70$ IU/L. Therefore the finding of a platelet count $>100,000 / \mathrm{uL}$ in a patient with very recent onset of epigastric pain and signs and symptoms consistent with a diagnosis of preeclampsia should not confuse the clinician into ruling out the probable diagnosis of HELLP syndrome. Importantly the finding of an AST level $\geq 70 \mathrm{IU} / \mathrm{L}$ was reliably present in all patients of this cohort even if the platelet count had not yet descended below 100,000/ul.

Liver hemorrhage and rupture can occur before platelets decrease below the $100,000 /$ ul threshold early in the development of HELLP Syndrome, consistent with the finding that some of our patient cohort with severe epigastric pain initially had platelets counts briefly above 100,000/ul. [19] Specifically, platelet counts obtained immediately preceding detection of a liver rupture/hematoma in the 9 women of our HELLP cohort with this complication were recorded at 167,000/ul, 109,000/ul, 80,000/ul, 78,000/ul, 51,000/ul, 49,000/ul, 24,000/ul, $20,000 / \mathrm{ul}$ and $17,000 / \mathrm{uL}$ always in association with epigastric pain. Thus platelet count per se does not help in determining likelihood of detectable liver bleeding or frank hepatic rupture nor does the sudden development of severe epigastric pain guarantee the presence or subsequent development of macroscopic hepatic hemorrhage or rupture. The potential use of routine hepatic ultrasound examination of patients with epigastric pain/HELLP syndrome probably deserves more study and physician consideration since it might facilitate liver integrity and injury evaluation in these mothers [20].

In our cohort of very ill HELLP patients with epigastric pain, it appears that intravenous magnesium sulfate alone was insufficiently effective monotherapy. Also, insufficiently rigorous control of severe systolic hypertension according to ACOG guidance since 2011 also is implicated in the high observed rate of stroke/ICH in our HELLP cohort [18] [21] [22]. Since a triple therapeutic approach of magnesium sulfate, blood pressure control and CORT has been advocated to constitute core therapeutic considerations for pregnant or postpartum patients with HELLP syndrome [15] [23], it was unexpected to discover that no patient in this series exhibiting severe symptomatology/severe epigastric pain, laboratory abnormalities and major maternal morbidity received CORT as a component of maternal therapy. It is noteworthy that the 8 patients in this series who did receive fetal lung maturation (FLM) steroids for preterm preeclampsia presentation did not flare into overt HELLP syndrome with epigastric pain until 12 hours to 4 days following receipt of the last FLM dose of betamethasone or 
dexamethasone.

We speculate that the absence of CORT use for maternal indications in these acutely ill HELLP women likely is relevant to the poor maternal outcomes observed. However, we acknowledge that there likely is selection bias present in any medicolegal series of patients inasmuch as the most seriously ill patients are more likely to be included in such studies involving small patient numbers. The longstanding institutional experience since the mid-1990's at the University of Mississippi Medical Center (UMMC) with CORT use initiated routinely and early for all HELLP patients (antepartum and postpartum) including those with epigastric pain is foundational to our speculation. A large prospective non-randomized cohort study of 190 consecutively treated patients with HELLP syndrome with or without epigastric pain between 2000-2007 employed a rigorously applied protocol including magnesium sulfate, strict blood pressure control and CORT therapy (intravenous dexamethasome $10 \mathrm{mg}$ IV every 12 hours for all patients) initiated immediately after a tentative or definitive diagnosis of HELLP was made [24]. There were no maternal deaths, no liver ruptures and no strokes. Moreover, two recent meta-analyses of CORT versus placebo [11] [12] found 1) significant improvement in HELLP laboratory markers, 2) less indicated blood transfusion and 3) shorter length of intensive care for CORT-treated HELLP patients.

Since mean blood pressure was also significantly reduced in the almost 800 CORT-treated patients compared to matched controls in the two recent meta-analyses [11] [12], it is possible that routine CORT use in our 42 HELLP cohort also might have reduced hypertension severity and stroke occurrence. The high incidence of stroke, liver injury, acute pulmonary edema and placental abruption likely is interrelated with uncontrolled SSH as well as the absence of CORT therapy in our HELLP cohort. The reported significant reduction in maternal blood pressure in CORT-treated mothers [11], especially severe elevations in blood pressure, likely is integral to risk reduction for stroke in HELLP patients treated with these medications [21] [25] [26].

\section{Summary}

In summary, HELLP Syndrome is an obstetric emergency which poses a significant threat to safe motherhood [5]. The findings summarized in the present report provide compelling evidence of the danger posed for pregnant and postpartum women by HELLP syndrome especially when manifested by severe epigastric pain. HELLP syndrome associated with sudden, severe epigastric pain appears to identify patients at very high risk to develop severe maternal morbidity and mortality [4]. Less than $100 \%$ utilization of magnesium sulfate and inadequate/ineffective antihypertensive therapy to control SSH are factors contributory to the very high maternal mortality and major maternal morbidity experienced by the 42 women in our cohort, likely aggravated by the absence of CORT therapy. Because significant liver pathology can be demonstrated very early in the development of HELLP syndrome soon after the beginning of severe 
epigastric pain, early initiation of CORT therapy likely is important before clinically evident brain, liver or other pathology develops [19] [26]. Prospective randomized clinical trials of various therapies including the early initiation of CORT for patients with HELLP syndrome, which do not distinguish between those with or without epigastric pain, may fail to identify benefit attributable to CORT. For the future it will be important to separate these two patient groups in order to improve our research and our practice.

\section{Confirmed}

Each author has confirmed compliance with the journal's requirements for authorship. The authors received no financial support for this undertaking. Neither has any potential conflict of interest. The first author prepared the original manuscript which the second author critiqued. This information has not been presented to a professional meeting nor is it under consideration for publication elsewhere.

\section{Conflicts of Interest}

The authors report no conflicts of interest.

\section{References}

[1] Weinstein, L. (1982) Syndrome of Hemolysis, Elevated Liver Enzymes, and Low Platelet Count: A Severe Consequence of Hypertension in Pregnancy. American Journal of Obstetrics and Gynecology, 142, 159-167. https://doi.org/10.1016/S0002-9378(16)32330-4

[2] Wallace, K., Harris, S., Addison, A. and Bean, C. (2018) HELLP Syndrome: Pathophysiology and Current Therapies. Current Pharmaceutical Biotechnology, 19, 816-826. https://doi.org/10.2174/1389201019666180712115215

[3] Ankumah, N.E. and Sibai, B.M. (2015) HELLP Syndrome: Clinical \& Laboratory Diagnosis. In: Martin Jr., J.N., Ed., The 2015 Compendium for HELLP Syndrome. From Bench to Bedside, Nova Science Publishers, Inc., New York, 19-28.

[4] Martin, J.N.Jr., Rinehart, B.K., May, W.L., Magann, E.F., Terrone, D.A. and Blake, P.G. (1999) The Spectrum of Severe Preeclampsia: Comparative Analysis by HELLP (Hemolysis, Elevated Liver Enzyme Levels, and Low Platelet Count) Syndrome Classification. American Journal of Obstetrics and Gynecology, 180, 1373-1384. https://doi.org/10.1016/S0002-9378(99)70022-0

[5] Martin Jr., J.N. (2013) The Howard Taylor Lecture: Milestones in the Quest for Best Management of Patients with HELLP Syndrome (Microangiopathic Hemolytic Anemia, Hepatic Dysfunction, Thrombocytopenia). International Journal of Gynecology \& Obstetrics, 121, 202-207. https://doi.org/10.1016/j.ijgo.2013.02.001

[6] Martin Jr., J.N., Brewer, J.M., Wallace, K., Sunesara, I., Canizaro, A., Blake, P.G., Lamarca, B. and Owens, M.Y. (2013) HELLP Syndrome and Composite Major Maternal Morbidity: Importance of Mississippi Classification System. The Journal of Maternal-Fetal \& Neonatal Medicine, 26, 1201-1206. https://doi.org/10.3109/14767058.2013.773308

[7] O’Brien, J.M. and Barton, J.R. (2005) Controversies with the Diagnosis and Management of HELLP Syndrome. Clinical Obstetrics and Gynecology, 48, 460-477. 
https://doi.org/10.1097/01.grf.0000160309.73197.35

[8] Vidaeff, A.C. and Yeomans, E.R. (2007) Corticosteroids for the Syndrome of Hemolysis, Elevated Liver Enzymes, and Low Platelets (HELLP): What Evidence? Minerva Obstetrics and Gynecology, 59, 183-190.

[9] Barton, J.R. (2015) HELLP Syndrome: Controversy over Corticosteroids. In: Martin Jr., J.N., Ed., The 2015 Compendium for HELLP Syndrome: From Bench to Bedside, Nova Science Publishers, Inc., New York, 63-75.

[10] Roberts, J.M., et al. (2013) Hypertension in Pregnancy: Report of the ACOG Task Force on Hypertension in Pregnancy. Obstetrics \& Gynecology, 122, 1122-1131. https://doi.org/10.1097/01.AOG.0000437382.03963.88

[11] Yang, L., Ren, C., Mao, M. and Cui, S. (2016) Prognostic Factors of the Efficacy of High-Dose Corticosteroid Therapy in Hemolysis, Elevated Liver Enzymes, and Low Platelet Count Syndrome during Pregnancy: A Meta-Analysis. Medicine, 95, e3203. https://doi.org/10.1097/MD.0000000000003203

[12] Mao, M. and Chen, C. (2015) Corticosteroid Therapy for Management of Hemolysis, Elevated Liver Enzymes, and Low Platelet Count (HELLP) Syndrome: A Meta-Analysis. Medical Science Monitor, 21, 3777-3783. https://doi.org/10.12659/MSM.895220

[13] Martin, J.N.Jr. and Tucker, J.M. (2020) Missing or Making the Timely Diagnosis of Acute Fatty Liver of Pregnancy (AFLP): Lessons Learned. The Journal of Maternal-Fetal \& Neonatal Medicine, 9, 1-7. https://doi.org/10.1080/14767058.2020.1832075

[14] Martin Jr., J.N. and Tucker, J.M. (2021) Maternal Morbidity and Mortality in Pregnant-Postpartum Women with Suspected HELLP Syndrome Identifiable as Probable TTP-aHUS by High LDH to AST Ratio. International Journal of Gynecology \& Obstetrics, 11.

[15] Martin, J.N.Jr. (2015) Core Concepts and Components of Best Management for Patients with HELLP Syndrome. In: Martin, J.N.Jr., Ed., the 2015 Compendium for HELLP Syndrome, Chapter 5, Nova Science Publishers, Inc., New York.

[16] Martin Jr., J.N., Bailey, A.P., Rehberg, J.F., Owens, M.T., Keiser, S.D. and May, W.L. (2008) Thrombotic Thrombocytopenic Purpura in 166 Pregnancies: 1955-2006. American Journal of Obstetrics and Gynecology, 199, 98-104. https://doi.org/10.1016/j.ajog.2008.03.011

[17] Keiser, S.D., Boyd, K.W., Rehberg, J.F., Elkins, S., Owens, M.Y., Sunesara, I. and Martin Jr., J.N. (2012) A High LDH to AST Ratio Helps to Differentiate Pregnancy-Associated Thrombotic Thrombocytopenic Purpura (TTP) from HELLP Syndrome. The Journal of Maternal-Fetal \& Neonatal Medicine, 25, 1059-1063. https://doi.org/10.3109/14767058.2011.619603

[18] ACOG Committee Opinion No. 767 (2019) Emergent Therapy for Acute-Onset, Severe Hypertension during Pregnancy and the Postpartum Period. Obstetrics \& Gynecology, 133, 409-412.

[19] Darby, M., Martin Jr., J.N., Mitchell, S.Q., Owens, M.Y. and Wallace, K. (2013) Using Case Reports to Determine When Liver Bleeding Occurs during Disease Progression in HELLP Syndrome. International Journal of Gynecology \& Obstetrics, 123, 7-9. https://doi.org/10.1016/j.ijgo.2013.06.007

[20] Perrone, L., Dohan, A., Bazeries, P., Guerrache, Y., Fohlen, A., Rousset, P., Aube, C., Laurent, V., Morel, O., Boudiaf, M., Hoeffel, C. and Soyer, P. (2015) Hepatic Involvement in HELLP Syndrome: An Update with Emphasis on Imaging Features. Abdominal Imaging, 40, 2839-2849. https://doi.org/10.1007/s00261-015-0481-1 
[21] Martin Jr., J.N.., Thigpen, B.D., Moore, R.C., Rose, C.H., Cushman, J. and May, W. (2005) Stroke and Severe Preeclampsia and Eclampsia: A Paradigm Shift Focusing on Systolic Blood Pressure. Obstetrics \& Gynecology, 105, 246-254. https://doi.org/10.1097/01.AOG.0000151116.84113.56

[22] Judy, A.E., McCain, C.L., Lawton, E.S., Morton, C.H., Main, E.K. and Druzin, M.L. (2019) Systolic Hypertension, Preeclampsia-Related Mortality, and Stroke in California. Obstetrics \& Gynecology, 133, 1151-1159. https://doi.org/10.1097/AOG.0000000000003290

[23] Martin Jr., J.N., Thigpen, B.D., Rose, C.H., Cushman, J., Moore, A. and May, W.L. (2003) Maternal Benefit of High-Dose Intravenous Corticosteroid Therapy for HELLP Syndrome. American Journal of Obstetrics and Gynecology, 189, 830-834. https://doi.org/10.1067/S0002-9378(03)00763-4

[24] Martin Jr., J.N., Owens, M.Y., Keiser, S.D., Parrish, M.F., Tam, K.B., Brewer, J.M., Cushman, J.L. and May, W.L. (2012) Standardized Mississippi Protocol Treatment of 190 Patients with HELLP Syndrome: Slowing Disease Progression and Preventing New Major Maternal Morbidity. Hypertension in Pregnancy, 31, 79-90. https://doi.org/10.3109/10641955.2010.525277

[25] Shields, L.E., Wiesner, S., Klein, C., Pelletreau, B. and Hedriana, H.L. (2017) Early Standardized Treatment of Critical Blood Pressure Elevations Is Associated with a Reduction in Eclampsia and Severe Maternal Morbidity. American Journal of $\mathrm{Ob}$ stetrics \& Gynecology, 216, 415.E1-415.E5. https://doi.org/10.1016/j.ajog.2017.01.008

[26] Wallace, K., Martin Jr., J.N., Tam, K., Wallukat, G., Dechend, R., Lamarca, B. and Owens, M.Y. (2013) Seeking the Mechanism(s) of Action for Corticosteroids in HELLP Syndrome: SMASH Study. American Journal of Obstetrics \& Gynecology, 208, 380.E1-380.E8. https://doi.org/10.1016/j.ajog.2013.01.049 